\title{
Outcome of the modified Dunn procedure in severe chronic or acute on chronic slipped capital femoral epiphysis
}

Nicola Ebert ${ }^{1,2+}$, Martin Rupprecht ${ }^{1,2+}$, Ralf Stuecker ${ }^{1,2}$, Sandra Breyer ${ }^{1,2}$, Norbert Stiel ${ }^{1,2}$, Matthias H. Priemel ${ }^{3}$ and Alexander S. Spiro ${ }^{1,2^{*}}$

\begin{abstract}
Background: In recent years, the modified Dunn osteotomy has gained popularity to treat slipped capital femoral epiphysis (SCFE) with various complication rates. Most studies included patients with different severities. This study aimed to determine (1) the radiological and clinical outcome, (2) the health-related quality of life, and (3) the incidence of avascular necrosis of the femoral head (AVN) in patients with severe chronic or acute on chronic SCFE treated by the modified Dunn procedure.

Methods: Out of 150 patients with SCFE treated at our institution between 2001 and 2014, 15 patients (mean age 12.9 years (range 11.8-15)) were treated by the modified Dunn procedure. Eight SCFE were chronic and 7 acute on chronic. All slips were severe with a mean Southwick slip angle (SSA) of $67^{\circ}$ (range 60-80). Radiographic and clinical outcomes were measured. Mean time of follow-up was 3.8 years (range 1-10).

Results: Anatomical reduction was achieved in all cases. Good radiological results according to the Stulberg Classification (grade $1+2$ ) and the Sphericity Deviation Score $(<30)$ were found in 9 out of 13 patients at the last follow-up. Clinical and functional outcome analysis revealed good results in 8 out of 10 patients (Harris Hip Score > 80). The quality of life measured by the Nottingham Health Profile (NHP) was described good in 10 out of 10 patients. Four out of 15 patients developed an AVN.

Conclusions: The modified Dunn procedure has a great potential to restore proximal femur geometry in severe chronic or acute on chronic SCFE. It should be considered only if there is no other possibility to restore proximal femur geometry, as is the case in severe slips, due to the risk of AVN.
\end{abstract}

Keywords: Modified Dunn procedure, Slipped capital femoral epiphysis, Avascular necrosis of the femoral head

\section{Introduction}

Up to date, there is still no consensus on how to treat slipped capital femoral epiphysis (SCFE). Complications after SCFE range from most severe avascular necrosis $(\mathrm{AVN})$ of the femoral head to metaphyseal deformity which may lead to femoroacetabular impingement and cartilage as well as labral damage [1-5]. Every SCFE

\footnotetext{
* Correspondence: aspiro@uke.de

${ }^{\dagger}$ Nicola Ebert and Martin Rupprecht contributed equally to this work and therefore share first authorship.

'Department of Pediatric Orthopaedics, Altonaer Children's Hospital,

Bleickenallee 38, 22763 Hamburg, Germany

${ }^{2}$ Department of Orthopaedics, University Medical Center

Hamburg-Eppendorf, Martinistraße 52, 20246 Hamburg, Germany

Full list of author information is available at the end of the article
}

should be stabilized to avoid further slipping and major complications, but further treatment depends on the type of SCFE. Different classification systems describe the severity of SCFE and the risk for AVN. Three classification systems are widely accepted, based on the stability of the slip [6], the duration of symptoms [7], and the extension of the slip [8].

In the treatment of acute, unstable slips, when weightbearing is not possible, there is an agreement that SCFE has to be surgically treated as an emergency case within $24 \mathrm{~h}[6,9,10]$. Whether additional hip decompression is a protective factor against AVN is not clear [11, 12].

In stable and chronic SCFE the treatment depends on the severity of the slip. For mild slips (Southwick angle

(C) The Author(s). 2019 Open Access This article is distributed under the terms of the Creative Commons Attribution 4.0 International License (http://creativecommons.org/licenses/by/4.0/), which permits unrestricted use, distribution, and 
$\left.<30^{\circ}\right)$ in situ pinning as final treatment has a wide acceptance as the prognosis, and the potential for remodeling of the metaphyseal deformity is believed to be good $[13,14]$. Moderate and severe slips have an increased risk of developing osteoarthritis [15]. After primary in situ stabilization, remodeling is not sufficient and residual metaphyseal deformity often leads to severe femoroacetabular impingement, cartilage as well as labral damage, and some shortening of the leg $[1,16]$. Many techniques have been described to correct this residual deformity. Intertrochanteric osteotomies are often used in moderate slips, but the potential for correcting the deformity is limited especially for severe slips [17].

For severe SCFE realigning, the deformity by a subcapital Dunn osteotomy by surgical hip dislocation has gained popularity [18-21]. There is a high potential to correct the deformity, but the potential for complications is significant. Among all complications, the incidence of AVN is of particular interest. Historical studies on subcapital wedge osteotomies have reported AVN rates of up to $54 \%[15,22]$. The modified Dunn procedure, where the retinacular vessels are protected in a periostal flap during the reduction of the femoral head has a lower AVN rate [19]. A review by Ziebarth and colleagues demonstrated no AVN in 40 hips treated by the modified Dunn procedure in two institutions [19]. Many subsequent studies reported AVN rates between 10 and $28 \%$ [20, 23, 24]. Comparing those studies is difficult. The classification systems used to describe the type of SCFE are different or unclear. Different types of SCFE, i.e., stable and unstable SCFE were combined in one study. Many studies are multicenter studies with different surgeons and treatment algorithms.

The purpose of this retrospective study was to determine (1) the radiological and clinical outcome, (2) the health-related quality of life, and (3) the incidence of avascular necrosis of the femoral head (AVN) in patients with severe chronic and acute on chronic SCFE of $>60^{\circ}$ treated by the modified Dunn procedure.

\section{Methods}

\section{Patients}

Out of 150 patients with SCFE treated at our institution between 2001 and 201415 patients fulfilled the inclusion criteria of having chronic or acute on chronic slips with a slip angle of $>60^{\circ}$ being treated by the modified Dunn procedure $[7,8]$. Patients who had previous surgery to correct hip deformity were excluded. All patients presented with pain and severe limping. Radiographs (anteroposterior and Lauenstein frog-leg view) were evaluated before and directly after surgery, as well as at the time of last follow-up [8]. The modified Dunn technique was elected to treat the deformities in order to restore femoral anatomy. All patients had surgery by the senior author (R.S.). Postoperative management included non-weight bearing for 8 weeks. Full weight-bearing was generally allowed after 12 weeks unless signs of avascular necrosis were detected. In these cases, non-weight bearing was recommended. Fifteen patients $(7$ males, and 8 females) could be included in the study. The mean age at operation was 12.9 years (range $11.8-15$ years). Mean follow-up was 3.8 years (range 1-10 years). Eight SCFE were chronic and 7 acute on chronic. All slips were severe with a mean SSA of $67^{\circ}$ (range $60-80^{\circ}$ ) (Table 1).

\section{Surgical procedure and postoperative protocol}

The stability of SCFE was evaluated during surgery in each case. SCFE was classified as unstable in case of a visible and demonstrable mobility between the metaphysis and epiphysis [25]. All patients had stable SCFE in this study. The surgical technique was according to the description of Ganz and colleagues [19]. The patient was positioned in a full lateral position, a trochanteric flip osteotomy was performed, a Z-shaped capsulotomy was done, and the femoral head was temporarily fixed with a K-wire, followed by surgical hip dislocation. The blood flow of the femoral head was tested by drilling with a Kwire before hip dislocation and after reduction in each case. The most important step was the preparation of a retinacular flap by which the retinacular vessels were protected. Further steps were the removal of the remaining physis and of abundant posterior callus. After osteotomy at the level of the physis, the head could be dissected off the femoral neck carefully, removed,

Table 1 Patient data

\begin{tabular}{|c|c|c|c|c|c|c|}
\hline & Age (year) & Sex & Clas & SSA & BMI $\left(\mathrm{kg} / \mathrm{m}^{2}\right)$ & ToFU (year) \\
\hline 1 & 12 & $F$ & C & $60^{\circ}$ & & 2 \\
\hline 2 & 12 & M & $\mathrm{AOC}$ & $65^{\circ}$ & 29 & \\
\hline 3 & 13 & M & $\mathrm{AOC}$ & $78^{\circ}$ & 33 & 10 \\
\hline 4 & 12.5 & M & $A O C$ & $60^{\circ}$ & 34 & 8.6 \\
\hline 5 & 13.7 & $\mathrm{~F}$ & $A O C$ & $70^{\circ}$ & 28 & 8 \\
\hline 6 & 12.4 & F & C & $78^{\circ}$ & 29 & 8 \\
\hline 7 & 13.6 & M & $\mathrm{AOC}$ & $60^{\circ}$ & & 1 \\
\hline 8 & 13.7 & M & $\mathrm{AOC}$ & $68^{\circ}$ & & 1 \\
\hline 9 & 15 & M & C & $80^{\circ}$ & 19 & 3 \\
\hline 10 & 12.9 & F & & $60^{\circ}$ & & \\
\hline 11 & 13 & $F$ & $A O C$ & $75^{\circ}$ & 31 & 3 \\
\hline 12 & 12.5 & $F$ & C & $60^{\circ}$ & 32 & 2 \\
\hline 13 & 11.8 & $F$ & & $60^{\circ}$ & 22 & 1 \\
\hline 14 & 12 & M & C & $70^{\circ}$ & 27 & 1.3 \\
\hline 15 & 13.5 & $F$ & C & $65^{\circ}$ & 28 & 1 \\
\hline Mean (SD) & $12.9(0.87)$ & & & $67^{\circ}\left(7.5^{\circ}\right)$ & $28(4.7)$ & $3.8(3.4)$ \\
\hline
\end{tabular}

Age age at time of surgery in years, $F$ female, $M$ male, Clas Classification, $C$ chronic, $A O C$ acute on chronic, SSA Southwick slip angle, $B M I$ body mass index, ToFU time of follow-up in years 
repositioned, and fixed with two 6.0-mm cannulated screws. Replacement and fixation of the greater trochanter was performed with two 4.5 or $6.5 \mathrm{~mm}$ screws. Postoperative hip flexion was limited to $70^{\circ}$, and an abduction splint was used while sitting or lying.

\section{Outcome measurements}

Assessment of radiographic outcome was done by analyzing the anteroposterior and Lauenstein frog-leg views preoperatively and at the last follow-up. The incidence of AVN, Stulberg Classification, and Sphericity Deviation Score was determined. The Stulberg Classification grades the congruency of the acetabulum and the femoral head [26]. Stulberg grades 1 and 2 are accepted to represent a good result, while grade 3 and more are considered to be non-satisfactory [27].

Sphericity Deviation Score is a measure for the sphericity of the femoral head [28]. A score up to 10 represents a very good, up to 30 a fair, and over 30 a nonsatisfactory result.

The complications are listed and graded by the DindoClavien Classification. Grade I complications require no treatment, grade II complications require other than usual postoperative treatment, grade III complications require surgical intervention, grade IV complications can lead to permanent disability, and grade $\mathrm{V}$ complication is fatal [29].

The clinical and functional outcome was assessed using the Harris Hip Score at the most recent follow-up [30]. Health-related quality of life was measured by the Nottingham health profile [31, 32]. Pain level was described by a visual analogue scale [33].

\section{Results}

\section{Radiographic measurements}

Anatomical reduction was achieved in all cases. Two patients could not be personally examined at the last follow-up, and radiographs of those two patients were not available. However, the available radiographic reports of both patients did not describe any signs of AVN after 12 and 20 months, respectively. Of the remaining 13 patients, 4 patients had an AVN, and 69\% (9 patients) had good results according to the Stulberg Classification and the Sphericity Deviation Score (Table 2) (Fig. 1).

\section{Clinical outcome and health-related quality of life}

Five patients were lost to clinical follow-up due to age ( $>18$ years of age at the time of FU) or they moved far away and were not available for further examination. One of them had an AVN. Regarding the remaining 10 patients, 8 patients $(80 \%)$ had good results in the Harris Hip Score.
Table 2 Outcome at last time of follow-up

\begin{tabular}{llllllll}
\hline & SC & SDS & HHS & NHP & VAS & AVN & DCC \\
\hline 1 & 2 & 7 & & & & & 0 \\
2 & & & & & & & II \\
3 & 5 & 44 & 58 & 0.97 & 0.8 & 1 & IV \\
4 & 2 & 28 & 90 & 0.8 & 2 & & 0 \\
5 & 2 & 29 & 80 & 0.79 & 1.5 & & 0 \\
6 & 3 & 78 & 77 & 0.93 & 6.2 & 1 & III \\
7 & 4 & 54 & & & & 1 & III \\
8 & 2 & 17 & & & & & $\|$ \\
9 & 2 & 21 & 96 & 0.9 & 0 & & 0 \\
10 & & & & & & & 0 \\
11 & 3 & 42 & 84 & 0.98 & 0.6 & 1 & II \\
12 & 2 & 19 & 93 & 0.98 & 0 & & 0 \\
13 & 1 & 11 & 100 & 0.96 & 0 & & 0 \\
14 & 2 & 8 & 86 & 0.86 & 1 & & 0 \\
15 & 1 & 3 & 93 & 0.91 & 2 & & 0 \\
Mean (SD) & & 27.8 & 85.7 & 0.91 & 1.6 & \\
& & $(21.7)$ & 12.1 & $(0.07)$ & $(1.7)$ &
\end{tabular}

SC Stulberg Classification, SDS Sphericity Deviation Score, HHS Harris Hip Score, NHP Nottingham Health Profile, VAS visual analog scale, AVN avascular hip necrosis, DCC Dindo-Clavien Classification

All 10 had good results in the Nottingham health profile. Only one out of 10 patients had relevant pain during daily activities.

\section{Complications}

At last follow-up, 4 of 15 patients (26\%) had developed AVN of the femoral head (Fig. 2). Three of them were acute on chronic slips, and they all occurred during the early phase of our study (Table 2).

In total, we found complications in 6 out of 15 patients (36\%). These patients had a DDC Score of II, III, or IV. As revision procedures, two early screw removals in patients with AVN followed by drilling of the necrotic bone were performed. Two patients had a leg length discrepancy which made an orthotic treatment necessary, and one of those patients had an AVN. Two patients, both treated in the early phase of our study, had joint instability (hip subluxation) after the modified Dunn procedure. One of them was treated by closed reduction and hip immobilization in a pelvic cast for 3 weeks. This patient developed AVN and had arthrodiastasis using an external fixation system 10 months after the modified Dunn procedure. The other patient had an open reduction with the removal of a loose body in the hip joint for the treatment of hip instability. An abduction splint was applied after surgery in this case.

\section{Discussion}

Our results demonstrate that anatomic reconstruction is possible also in the most severe forms of SCFE if treated 



Fig. 1 Preoperative $(\mathbf{a}, \mathbf{b})$ and 1 year postoperative $(\mathbf{c}, \mathbf{d})$ X-rays of a 13-year-old female patient with an acute on chronic SCFE with an SSA of $65^{\circ}$

by the modified Dunn procedure. In $70 \%$ of the patients, the radiographic outcome is satisfactory or good, and the clinical outcome is even better, with $80 \%$ satisfactory results. Patients are less affected in quality of life even when radiographic and functional results were less satisfactory. There were also significant complications like AVN in 4 patients. We cannot finally say whether AVN occurred because of surgery or because of the slip itself because we did not perform perfusion MRI before and after surgery. The blood flow was tested before hip dislocation and after reduction by drilling of the femoral head with a K-wire, but this is not a safe method to ensure head perfusion. Upasani reported that the intraoperative assessment of head perfusion did not correlate with postoperative outcome [34]. Slongo, Ziebarth, and Huber described that the clinical stability of SCFE does not correlate with intraoperative stability [20, 25, 35]. The rate of AVN in our study is comparable to previous 

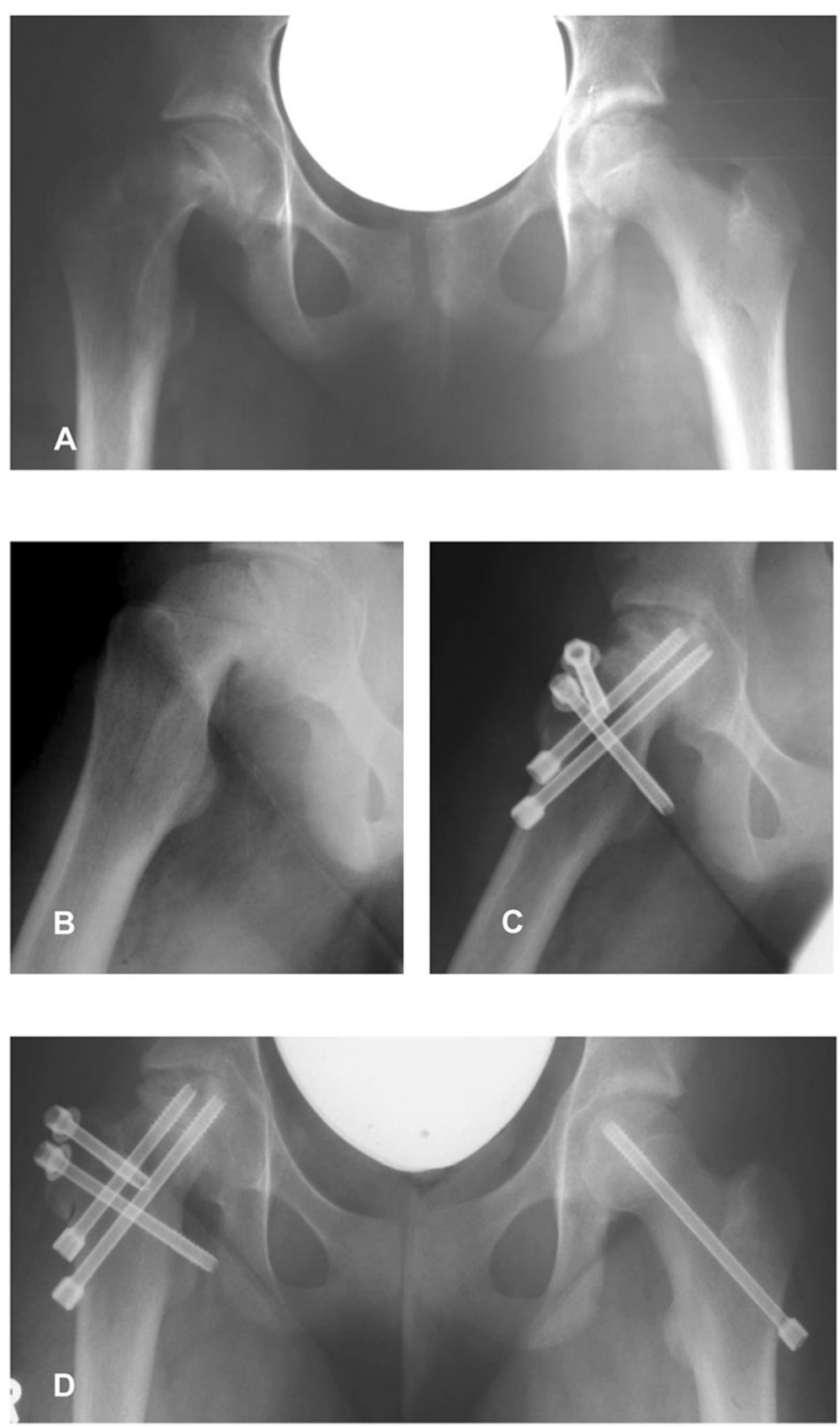

Fig. 2 Preoperative $(\mathbf{a}, \mathbf{b})$ and 1 year postoperative $(\mathbf{c}, \mathbf{d})$ X-rays of a 12-year-old female patient with an acute on chronic SCFE with an SSA of 78 ${ }^{\circ}$ This patient developed a partial avascular necrosis of the femoral head

case series. However, in most studies, slips of various magnitudes were analyzed making comparisons with this study, including severe slips only, difficult. Most studies had lower AVN rates. Ziebarth and colleagues had no AVN in their study group with a follow-up of 1-3 years [19]. But Upasani et al. reported a complication rate of $37 \%$ and an AVN rate of $23 \%$ in their mixed patient cohort with a mean follow-up of 2.6 years [34]. In unstable slips, Sankar et al. noted an AVN rate of $26 \%$ after 22 months of follow-up [24]. The most common complication was revision surgery due to implant-related complications $[19,20,33]$. In our case series, those complications were not encountered. By now, arthrodiastasis using an external fixation system for 4 months combined with the drilling of the AVN region is usually used to treat AVN at our institution. These patients were mobilized with non-weight bearing of the affected hip for 6 months. 
The modified Dunn procedure has also been advocated for unstable slips. Huber et al. outline the advantage of direct evaluation of epiphyseal perfusion during the procedure [35]. Tibor and Sink also suggest the modified Dunn procedure to be a good treatment option for acute and unstable slips [36]. Nevertheless, they reported a higher potential for complications than pinning in situ and a steep learning curve for the surgeon [36]. Sucato et al. recently stated in their review of patients treated by surgical hip dislocation that the average AVN rate for in situ pinning is $23.9 \%$ and for the modified Dunn procedure $16.7 \%$ when all studies looking at unstable SCFE are pooled [37]. Parsch et al. reported an AVN rate of $4.7 \%$ in their series of 64 patients with unstable slips, which were treated by capsulotomy, gentle reduction, and K-wire pinning [11].

The modified Dunn procedure can restore proximal femoral anatomy and hip function as shown in our study. Additionally, the intraarticular pathology can be evaluated and addressed at the time of surgery. Concerning the high rate of severe complications, we also use other surgical procedures for moderate stable slips, e.g., the Imhäuser osteotomy. Bali recently showed good results by combining the Imhäuser osteotomy with a femoral neck osteoplasty [38]. For the SCFE with a SSA greater than $50^{\circ}$, the intertrochanteric osteotomies should not be used, because of inacceptable postoperative deformity $[1,39]$.

The Dunn procedure is a good treatment option for those patients with severe chronic or acute on chronic slips. We saw that most of our AVN complications occurred in the early phase of our study, and we anticipate that complications like AVN will probably decrease with more experience. As we did not use MRI before surgery, we cannot definitely say whether the blood flow of the femoral head was compromised due to the slip or due to surgery in patients who developed AVN.

The limitations of this study are certainly the small number of patients, the retrospective character, and a missing control group. Additionally, the time of followup is different for each patient.

\section{Conclusions}

The clinical and radiological outcome was good or satisfactory in most of the patients after modified Dunn procedure in this series. However, due to several limitations, conclusions have to be drawn with caution. Based on the results of this study, we believe that the modified Dunn procedure is only indicated if there is no other possibility to restore proximal femur geometry, as is the case in severe chronic or acute on chronic slipped capital femoral epiphysis. These slips cannot be repositioned and would lead to severe impingement, leg shortening, limited range of motion, and severe osteoarthritis $[2,9,15,40]$.

\section{Abbreviations}

AVN: Avascular necrosis; NHP: Nottingham Health Profile; SCFE: Slipped capital femoral epiphysis; SSA: Southwick slip angle

\section{Acknowledgements}

Not applicable.

\section{Authors' contributions}

NE has made substantial contributions to the conception and design of the work, acquisition, analysis, and interpretation of data and has drafted the work, approved the submitted version (and any substantially modified versions that involve the author's contribution to the study), and agreed both to be personally accountable for the author's own contributions and to ensure that questions related to the accuracy or integrity of any part of the work, even ones in which the author was not personally involved, are appropriately investigated, resolved, and the resolution documented in the literature. MR has made substantial contributions to the conception and design of the work, interpretation, and analysis of data and substantively revised the work, approved the submitted version (and any substantially modified versions that involve the author's contribution to the study), and agreed both to be personally accountable for the author's own contributions and to ensure that questions related to the accuracy or integrity of any part of the work, even ones in which the author was not personally involved, are appropriately investigated, resolved, and the resolution documented in the literature. RF has made substantial contributions to the conception and design of the work, interpretation, and analysis of data and substantively revised the work, approved the submitted version (and any substantially modified versions that involve the author's contribution to the study), and agreed both to be personally accountable for the author's own contributions and to ensure that questions related to the accuracy or integrity of any part of the work, even ones in which the author was not personally involved, are appropriately investigated, resolved, and the resolution documented in the literature. SB has made substantial contributions to the design of the work and interpretation of data and has approved the submitted version (and any substantially modified versions that involve the author's contribution to the study), and agreed both to be personally accountable for the author's own contributions and to ensure that questions related to the accuracy or integrity of any part of the work, even ones in which the author was not personally involved, are appropriately investigated, resolved, and the resolution documented in the literature. NS has made substantial contributions to the design of the work and interpretation of data and has approved the submitted version (and any substantially modified version that involves the author's contribution to the study) and has agreed both to be personally accountable for the author's own contributions and to ensure that questions related to the accuracy or integrity of any part of the work, even ones in which the author was not personally involved, are appropriately investigated, resolved, and the resolution documented in the literature. MHP has made substantial contributions to the interpretation of data, has approved the submitted version (and any substantially modified version that involves the author's contribution to the study), and has agreed both to be personally accountable for the author's own contributions and to ensure that questions related to the accuracy or integrity of any part of the work, even ones in which the author was not personally involved, are appropriately investigated, resolved, and the resolution documented in the literature. ASS has made substantial contributions to the conception and design of the work and interpretation of data and has substantively revised work, approved the submitted version (and any substantially modified version that involves the author's contribution to the study), and agreed both to be personally accountable for the author's own contributions and to ensure that questions related to the accuracy or integrity of any part of the work, even ones in which the author was not personally involved, are appropriately investigated, resolved, and the resolution documented in the literature.

\section{Funding}

The authors did not receive any outside funding, honorarium, grants, or other forms of payment in support of their research for or preparation of this work, and there are no conflicts of interest. 


\section{Availability of data and materials}

The data (references) used to support the findings of this study are included within the article.

\section{Ethics approval and consent to participate}

This is a retrospective study. Ethics approval is not necessary.

\section{Consent for publication}

Not applicable.

\section{Competing interests}

The authors declare that they have no competing interests.

\section{Author details}

'Department of Pediatric Orthopaedics, Altonaer Children's Hospital, Bleickenallee 38, 22763 Hamburg, Germany. ${ }^{2}$ Department of Orthopaedics, University Medical Center Hamburg-Eppendorf, Martinistraße 52, 20246 Hamburg, Germany. ${ }^{3}$ Department of Trauma, Hand, and Reconstructive Surgery, University Medical Center Hamburg-Eppendorf, Martinistraße 52, 20246 Hamburg, Germany.

Received: 26 July 2019 Accepted: 24 October 2019

Published online: 08 November 2019

\section{References}

1. Leunig M, Casillas MM, Hamlet M, Hersche O, Nötzli H, Slongo T, Ganz R. Slipped capital femoral epiphysis: early mechanical damage to the acetabular cartilage by a prominent femoral metaphysic. Acta Orthop Scand. 2000;71(4):370-5.

2. Ganz R, Huff TW, Leunig M. Extended retinacular soft tissue flap for intraarticular hip surgery: surgical technique, indications and results of application. Instr Course Lect. 2009;58:241-55.

3. Rebello G, Spencer S, Millis MB, Kim YJ. Surgical dislocation in the management of pediatric and adolescent hip deformity. Clin Orthop Relat Res. 2009;467(3):724-31.

4. Spencer S, Millis MB, Kim YJ. Early results of treatment of hip impingement syndrome in slipped capital femoral epiphysis and pistol grip deformity of the femoral head-neck junction using the surgical dislocation technique. J Pediatr Orthop. 2006;26(3):281-5.

5. Kennedy JG, Hresko MT, Kasser JR, Shrock KB, Zurakowski D, Waters PM, Millis MB. Osteonecrosis of the femoral head associated with slipped capital femoral epiphysis. J Pediatr Orthop. 2001;21(2):189-93.

6. Loder RT, Richards BS, Shapiro PS, Reznick LR, Aronson DD. Acute slipped capital femoral epiphysis: the importance of physeal stability. J Bone Joint Surg Am. 1993;75(8):1134-40

7. Fahey JJ, O'Brien ET. Acute slipped capital femoral epiphysis: review of the literature and report of ten cases. J Bone Joint Surg Am. 1965;47:1105-27.

8. Southwick WO. Osteotomy through the lesser trochanter for slipped capital femoral epiphysis. J Bone Joint Surg Am. 1967;49(5):807-35.

9. Tokmakova KP, Stanton RP, Mason DE. Factors influencing the development of osteonecrosis in patients treated for slipped capital femoral epiphysis. J Bone Joint Surg Am. 2003;85(5):798-801.

10. Peterson MD, Weiner DS, Green NE, Terry CL. Acute slipped capital femoral epiphysis: the value and safety of urgent manipulative reduction. J Pediatr Orthop. 1997:17(5):648-54.

11. Parsch K, Weller S, Parsch D. Open reduction and smooth Kirschner wire fixation for unstable slipped capital femoral epiphysis. J Pediatr Orthop. 2009;29(1):1-8.

12. Ibrahim T, Mahmoud S, Riaz M, Hegazy A, Little DG. Hip decompression of unstable slipped capital femoral epiphysis: a systematic review and metaanalysis. J Child Orthop. 2015;9(2):113-20.

13. Jones JR, Paterson DC, Hillier TM, Foster BK. Remodelling after pinning for slipped capital femoral epiphysis. J Bone Joint Surg (Br). 1990;72(4):568-73.

14. Engelhardt P. Natural course of epiphysiolysis of the femur head. Orthopade. 1994;23(3):195-9

15. Carney BT, Weinstein SL. Natural history of untreated chronic slipped capital femoral epiphysis. Clin Orthop Relat Res. 1996;322:43-7.

16. Beck M, Kalhor M, Leunig M, Ganz R. Hip morphology influences the pattern of damage to the acetablar cartilage. Femuroacetabular impingement as cause of early osteoarthritis of the hip. J Bone Joint Surg (Br). 2005;87(7):1012-8.
17. Witbreuk M, Bolkenbaas M, Mullender MG, Sierevelt IN, Besselaar PP. The results of downgrading moderate and severe slipped capital femoral epiphysis by an early Imhauser femur osteotomy. J Child Orthop. 2009;3(5): 405-10

18. Ganz R, Leunig M, Leunig-Ganz K, Harris WH. The etiology of osteoarthritis of the hip: an integrated mechanical concept. Clin Orthop Relat Res. 2008, 466(2):264-72.

19. Ziebarth K, Zilkens C, Spencer S, Leunig M, Ganz R, Kim YJ. Capital realignment for moderate and severe SCFE using a modified Dunn procedure. Clin Orthop Relat Res. 2009;467(3):704-16.

20. Slongo T, Kakaty D, Krause F, Ziebarth K. Treatment of slipped capital femoral epiphysis with a modified Dunn procedure. J Bone Joint Surg Am. 2010:92(18):2898-908.

21. Dunn DM. The treatment of adolescent slipping of the upper femoral epiphysis. J Bone Joint Surg Br. 1964:46:621-9.

22. Dunn DM, Angel JC. Replacement of the femoral head by open operation in severe adolescent slipping of the upper femoral epiphysis. J Bone Joint Surg (Br). 1978;60-B(3):394-403.

23. Lawane M, Belouadah M, Lefort G. Severe slipped capital femoral epiphysis: the Dunn's operation. Orthop Traumatol Surg Res. 2009;95(8):588-91.

24. Sankar WN, Vanderhave KL, Matheney T, Herrera-Soto JA, Karlen JW. The modified Dunn procedure for unstable slipped capital femoral epiphysis: a multi center perspective. J Bone Joint Surg Am. 2013;95(7):585-91.

25. Ziebarth K, Domayer S, Slongo T, Kim YJ, Ganz R. Clinical stability of slipped capital femoral epiphysis does not correlate with intraoperative stability. Clin Orthop Relat Res. 2012:470:2274-12

26. Stulberg D, Cooperman DR, Wallenstein R. The natural history of LeggCalvé-Perthes disease. J Bone Joint Surg Am. 1981;63(7):1095-108.

27. Wiig $\mathrm{O}$, Terjesen $\mathrm{T}$, Svenningsen $\mathrm{S}$. Inter-observer reliability of the Stulberg classification in the assessment of Perthes disease. J Child Orthop. 2007;1 (2): 101-5.

28. Siddesh ND, Shah H, Tercier S, Pai H, Nair S, Joseph B. The sphericity deviation score: a quantitative radiologic outcome measure of Legg-Calvé Perthes disease applicable at the stage of healing and at skeletal maturity. J Pediatr Orthop. 2014:34(5):522-8.

29. Dindo D, Demartines N, Clavien PA. Classification of surgical complications: a new proposal with evaluation in a cohort of 6336 patients and results of a survey. Ann Surg. 2004;240(2):205-13.

30. Harris WH. Traumatic arthritis of the hip after dislocation and acetabular fractures: treatment by mold arthroplasty. An end-result study using a new method of result evaluation. J Bone Joint Surg Am. 1969;51(4):737-55.

31. Hunt SM, McEwen J, McKenna SP. Measuring health status: a new tool for clinicians and epidemiologists. J R Coll Gen Pract. 1985;35(273):185-8.

32. Kohlmann T, Bullinger M, Kirchberger-Blumstein I. German version of the Nottingham health profile (NHP): translation and psychometric validation. Soz Praventivmed. 1997:42(3):175-85.

33. Scott J, Huskisson E. Graphic representation of pain. Pain. 1976;2(2):175-84.

34. Upasani W, Matheney TH, Spencer SA, Kim YJ, Millis MB, Kasser JR. Complications after modified Dunn osteotomy for the treatment of adolescent slipped capital femoral epiphysis. J Pediatr Orthop. 2014; 34(7):661-7.

35. Huber H, Dora C, Ramseier LE, Buck F, Dierauer S. Adolescent slipped capital femoral epiphysis treated by a modified Dunn osteotomy with surgical hip dislocation. J Bone Joint Surg Br. 2011;93(6):833-8.

36. Tibor LM, Sink EL. Risks and benefits of the modified Dunn approach for treatment of moderate or severe slipped capital femoral epiphysis. J Pediatr Orthop. 2013:33(1):99-102.

37. Sucato DJ, de la Rocha A. High-grade SCFE: the role of surgical hip dislocation and reduction. J Pediatr Orthop. 2014:34(1):18-24.

38. Bali NS, Harrison SO, Bache CE. A modified Imhäuser osteotomy. An assessment of an addition of an open femoral neck osteoplasty. Bone and Joint J. 2014:96:1119-23.

39. Imhäuser G. Pathogenesis and therapy of hip dislocation in youth. Z Orthop und Grenzgeb. 1956;88:3-41.

40. Sink EL, Zaltz I, Heare T, Dayton M. Acetabular cartilage and labral damage observed during surgical hip dislocation for stable slipped capital femoral epiphysis. J Pediatr Orthop. 2010;30(1):26-30.

\section{Publisher's Note}

Springer Nature remains neutral with regard to jurisdictional claims in published maps and institutional affiliations. 